\title{
ERRATUM
}

\section{Erratum to: The Impact of Method, Motivation, and Empathy on Diversity Training Effectiveness}

\author{
Alex Lindsey • Eden King • Michelle Hebl • \\ Noah Levine
}

Published online: 29 November 2014

(c) Springer Science+Business Media New York 2014

\section{Erratum to: J Bus Psychol}

\section{DOI 10.1007/s10869-014-9384-3}

In Table 2 of the original publication, the numeric entries of the second row are not displayed appropriately. The correct version of Table 2 is presented as follows:

Table 2 Summary of multiple regression analysis

\begin{tabular}{|c|c|c|c|c|c|c|c|c|c|c|c|c|}
\hline \multirow[t]{3}{*}{ Independent variable } & \multicolumn{12}{|c|}{ Dependent variable } \\
\hline & \multicolumn{3}{|c|}{ ATLG } & \multicolumn{3}{|c|}{ LGB supportive behavior } & \multicolumn{3}{|c|}{ Modern racism } & \multicolumn{3}{|c|}{ Ethnic supportive behavio } \\
\hline & $B$ & $S E B$ & $\beta$ & $B$ & $S E B$ & $\beta$ & $B$ & $S E B$ & $\beta$ & $B$ & $S E B$ & $\beta$ \\
\hline Target of training & $-.50 *$ & .23 & $-.19 *$ & .30 & .20 & .14 & .14 & .16 & -.08 & .22 & .17 & .12 \\
\hline Goal setting & .56 & .30 & .20 & .19 & .25 & .08 & .21 & .20 & .11 & .02 & .22 & .01 \\
\hline Perspective taking & -.41 & .28 & -.15 & $.53 *$ & .24 & $.23 *$ & .03 & .20 & .02 & .09 & .21 & .05 \\
\hline$R^{2}$ & & & $.07 *$ & & & $.07 *$ & & & .02 & & & .02 \\
\hline
\end{tabular}

Target of training $(1=$ race, 2 = LGB), goal setting, and perspective taking were all dummy coded before being entered into the regression analysis

$* p<.05 ; * * p<.01$

The online version of the original article can be found under doi:10.1007/s10869-014-9384-3.

A. Lindsey $(\bowtie) \cdot$ E. King

George Mason University, 4400 University Drive, MSN 3F5,

Fairfax, VA 22030, USA

e-mail: alindse3@gmu.edu

M. Hebl · N. Levine

Rice University, Houston, USA 